\title{
LATINOAMERICANOS EN ESPAÑA: LA INTEGRACIÓN EN LA EUROPA DE LA EXCLUSIÓN SOCIAL
}

\author{
MIGUEL ÁNGEL MATEO PÉREZ \\ DANIEL LA PARRA \\ Departamento de Sociología II. Universidad de Alicante (España).
}

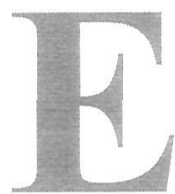

\section{RESUMEN}

ste trabajo se estructura en cuatro puntos generales. En primer lugar, se realiza una reflexión general sobre migraciones internacionales, enfoques teóricos y procesos de desarrollo, para, más tarde, realizar una aproximación al número y las características de los migrantes, en general, y a los latinoamericanos, en particular, residentes en España, atendiendo a su evolución en los últimos 10 años. En tercer lugar, se analizan las características de la inmigración y sus efectos sobre el mercado de trabajo español. Para concluir, en cuarto lugar, se exponen los factores de inclusión y exclusión social de los migrantes latinoamericanos en el contexto de la sociedad española.

Palabras clave: Migración internacional, integración, exclusión, latinos.

\section{MIGRACIONES INTERNACIONALES: ALGUNAS EXPLICA- CIONES}

El estudio de las migraciones internacionales y el desarrollo de las Ciencias Sociales se ha producido de forma paralela. Sin embargo, el trabajo sistemático sobre las migraciones como objeto de estudio no se produce hasta el siglo XIX cuando Ravestein enuncia sus bien conocidas "leyes". El estudio de las migraciones se ha producido desde diferentes prismas en un enriquecedor debate aún abierto sobre sus causas y consecuencias.

En este momento, la inexistencia de una teoría general plenamente satisfactoria que se tome como paradigma para el estudio de las migraciones es más una ventaja que un inconveniente. A nuestro juicio, 
la proliferación de investigaciones, redes de trabajo y equipos interdisciplinares ha permitido a las migraciones humanas su institucionalización como objeto de estudio especializado dentro de las ciencias sociales.

Ante la ausencia de un paradigma general sobre las migraciones internacionales, tres son las estrategias o heurísticos utilizados para la aproximación al estudio científico de las migraciones.

1) Abordar el conocimiento de las migraciones humanas realizando estudios de casos específicos, mucho más cercanos a la etnografía o a la sociografía que a la sociología propiamente dicha. En la actualidad, este enfoque es uno de los que más éxito han generado, produciéndose un gran número de estudios centrados principalmente en:

- análisis de colectivos concretos de inmigrantes (según ciudadanía, lugar de nacimiento, cultura, etnia, religión...),

- análisis de comunidades concretas de recepción (países, regiones, municipios, barrios),

- análisis de las dimensiones específicas del fenómeno migratorio (identidades colectivas, racismo, impactos sobre el mercado de trabajo, situación de las mujeres migrantes, etc.),

- análisis de las conjunciones de elementos anteriores: análisis de las condiciones de trabajo de un determinado colectivo de migrantes en un municipio o de las pautas de fecundad de determinadas mujeres migrantes en un enclave específico.

Todos estos análisis tienen como denominador común la producción de información empírica (datos) tanto cualitativos como cuantitativos, pero difícilmente se encuentran articulados los resultados de investigación como para producir efectos políticos.

2) La segunda estrategia de investigación, también muy abundante en la actualidad, consiste en el análisis de datos cuantitativos procedentes de las bases de datos estadísticas existentes. La mayor o menor pericia en el análisis estadístico, además de los problemas sabidos de validez y fiabilidad de los datos producidos por ciertas instituciones, que parten de los problemas de definición del concepto de migración hasta errores clamorosos en la medición en cuestionarios de censos y/o padrones o de encuestas a colectivos específicos, complican esta opción. Sin embargo, el análisis de flujos, los intentos por medir exactamente los stocks y/o las características sociodemográficas de los migrantes e incluso añadir la perspectiva longitudinal en 
las investigaciones cuantitativas, los cada vez más frecuentes en una literatura académica y en contextos de investigación comparada.

3) La tercera estrategia de investigación es aquélla que exclusivamente hace referencia a investigaciones teóricas. Ciertamente, el trabajo teórico sobre migraciones que no se apoya en investigación empírica es cada vez más escaso. Pero aún existe toda una producción académica que aborda sin contacto empírico con la realidad aspectos parciales del fenómeno migratorio (esto es, toman como núcleo de las reflexiones una dimensión del procesos migratorio, habitualmente sus causas o sus consecuencias), dando como resultado un conjunto de teorías de grado medio (a las que se refiriera con acierto Merton) que de ninguna manera pueden combinarse o estructurarse de teorías generales

Estas teorías de grado o alcance medio son las que han dado origen a las clasificaciones más conocidas, las realizadas por Portes y Bach en 1985 y la de Massey, Arango, Pellegrino y otros ya en la década de los 90.

A grandes rasgos, estas clasificaciones de las teorías de grado medio abarcan desde los postulados de la economía clásica del siglo XIX y primera mitad del siglo XX, con aportaciones que llegan hasta nuestros días en las teorías de "atracción-expulsión" (push-pull) hasta las más recientes ya en la segunda mitad del siglo XX. Sin detenernos demasiado, veamos una perspectiva general de estas teorías, tal y como se puede observar en el cuadro 1.

Como en otros enfoques de tipo estructural no funcionalista, se plantea que los movimientos de población se producen desde los países del sur (o periféricos) hacia los países del norte (o centrales), provocados por una serie de desigualdades estructurales o desequilibrios económicos (y de desarrollo) mundiales. Estos desequilibrios son producidos básicamente por la penetración del capitalismo como modo de producción en los países periféricos y acelerados en los últimos tiempos por la mundialización de la economía y la globalización de la vida social, que parece ha beneficiado de manera desigual a sus actores en función, a mi juicio, de su posición en la cadena de mercancías mundial.

La migración, entonces, se puede interpretar como un efecto más de la dominación ejercida por parte de las zonas centrales del sistema mundial sobre las periféricas en un contexto de relaciones internacionales en las que los conflictos de intereses son patentes. Estos conflictos entre centro (expansivo) y periferia (fragmentada, penetrada cultural y económicamente) provocan movimientos de población de la periferia al centro contribuyendo aún más al desequilibrio mundial, ya que las 
posibilidades reales de mejora en términos estructurales de las zonas emigrantes son muy reducidas, precisamente por su debilidad en la estructura productiva mundial.

Cuadro 1. Principalles teorías migratorias desde la segunda mitad dell siglo $\mathbf{X X}$

\begin{tabular}{|c|c|}
\hline Dimensión & Clasificación de Massey y otros + Portes y Bach \\
\hline \multirow{5}{*}{$\begin{array}{l}\text { INICIO / CAUSAS DEL } \\
\text { MOVIMIENTO MIGRATORIO }\end{array}$} & $\begin{array}{l}\text { Economía neoclásica (Macro-Micro) o Teoría del Mercado } \\
\text { de Trabajo } \\
\text { TODARO-BORJAS }\end{array}$ \\
\hline & $\begin{array}{l}\text { Nueva Economía } \\
\text { STARK }\end{array}$ \\
\hline & $\begin{array}{l}\text { Teoría del Mercado dual } \\
\text { PRIORE }\end{array}$ \\
\hline & $\begin{array}{l}\text { Teorías de orientación marxista } \\
\text { CASTLES y KOSACK }\end{array}$ \\
\hline & $\begin{array}{l}\text { Teoría del Sistema Mundial } \\
\text { WALLERSTEIN }\end{array}$ \\
\hline \multirow{6}{*}{$\begin{array}{l}\text { MANTENIMIENTO DE LOS } \\
\text { MOVIMIENTOS MIGRATORIOS }\end{array}$} & $\begin{array}{l}\text { Teoría de las redes sociales } \\
\text { MASSEY }\end{array}$ \\
\hline & Teoría Institucional \\
\hline & Teoría de la causación acumulativa \\
\hline & MASSEY \\
\hline & $\begin{array}{l}\text { Teoría de los sistemas migratorios } \\
\text { ZLOTNIK }\end{array}$ \\
\hline & Teoría del proyecto migratorio \\
\hline \multirow{3}{*}{$\begin{array}{l}\text { FUNCIONES DE LAS } \\
\text { MIGRACIONES (EFECTOS } \\
\text { SOBRE LAS EONOMIAS) } \\
\end{array}$} & Teorías del consenso \\
\hline & Teorías del conflicto \\
\hline & Teorías del conflicto sostenido \\
\hline \multirow{3}{*}{$\begin{array}{l}\text { INTEGRACIÓN DE LOS } \\
\text { MIGRANTES (MODELOS) }\end{array}$} & Asimilación \\
\hline & Crisol de culturas (melting pot) \\
\hline & Pluralismo \\
\hline
\end{tabular}

Las empresas multi y transnacionales (incluidas las que tienen como domicilio fiscal la Unión Europea y países nada sospechosos como España), la inversión extranjera directa y las administraciones políticas estatales de los países periféricos caracterizadas por una debilidad estructural a la hora de negociar posiciones contrarias al flujo de capitales afectan directamente a la movilidad humana. Ciertamente, eso no significa que la motivaciones individuales no cuenten en el proceso migratorio. Además de éstas, hay que tener presente las cuestiones anteriormente mencionadas.

Para contrarrestar las pérdidas en la tasa de ganancia neta, los actores centrales en el sistema mundial intervienen en las periferias en busca de materias primas de toda índole y de mano de obra barata, formada y no sindicalizada. La sustitución de los modelos de desarrollo local en las periferias por otros en los que su posición es de clara desventaja produce desequilibrios económicos y sociales, entre los que se encuentra el desplazamiento de trabajadores y trabajadoras que no tienen espacios en sus ámbitos locales para realizar sus proyectos vitales. En algunos casos, siquiera satisfacer sus necesidades básicas. 
En este proceso de desvertebración de las periferias, la movilidad humana se produce primero del campo a la ciudad, dado el gran excedente de mano de obra desplazada desde el sector agrario, creándose en las ciudades un sector terciario (servicios), de una productividad extremadamente baja, predispuesto a la migración internacional utilizando los mismos canales que se abrieron en la penetración económica y generaron nexos culturales entre los centros y las periferias.

En los países centrales, los migrantes desemplean actividades económicas en sectores que se apoyan en una mano de obra barata para poder mantener una tasa de beneficios elevada. En palabras de Saskia Sassen, la migración funciona como un sistema mundial de suministro de mano de obra, es decir, como un sistema de transferencia de lo más precioso y preciado en los procesos de desarrollo desde las periferias: el capital humano.

Si estamos más o menos de acuerdo con que un enfoque posible para explicar la movilidad humana en el actual contexto histórico algo tiene con relaciones estructurales entre actores del centro, periferia y semiperiferia (como España, por ejemplo) podemos convenir que una de las alternativas es la redefinición de los modelos de desarrollo conscientes que es posible una organización alternativa de la producción y distribución de bienes y servicios centrada en la satisfacción de las necesidades humanas básicas.

\section{EXTRANJEROS Y MIGRANTES EN ESPAÑA}

En el contexto de la UE, los flujos migratorios han sido intensos durante toda la década de los 90 y especialmente intensos en lo que llevamos de ésta. No en vano, desde el año 2000, los saldos migratorios netos anuales superaron el millón y medio de personas. En el 2002, más de trece millones de extranjeros nacionales de terceros países residían en la UE, lo que vendría a suponer, aproximadamente, un 3,4\% de la población.

Quizá lo más llamativo sea el cambio de las áreas de origen y destino. Los países que fueron grandes receptores de inmigración durante la década de los 60 y 70 (Francia, Reino Unido y Alemania) han sido sustituidos por los países del Mediterráneo y sur de Europa. España, Italia y Portugal acogieron a la mitad del saldo migratorio neto de toda la UE en 2003. La contribución de España a la migración neta en la EU fue del 23\%, la de Italia fue del $21 \%$ y la de Portugal del 6\%.

En España, de aproximadamente 350.000 extranjeros censados en 1991 hemos pasado a 1.500.000 en el censo de 2001 y, según los datos 
del Padrón Municipal de Habitantes, para 2003 las cifras están rondando casi los 2.700 .000 extranjeros, lo que implica un $6,2 \%$ del total de empadronados frente al 1,4\% de 1996.

La perspectiva de la Unión Europea recientemente ampliada plantea interrogantes sobre los flujos de migrantes de Europa Central y Oriental que dejarán de ser extracomunitarios al aplicarse la libre circulación de personas y trabajadores. Sin embargo, el Tratado de Adhesión permite a los Estados miembros mantener una moratoria de entre dos y cinco años a la aplicación de la libre circulación de personas y trabajadores, precisamente con el objetivo de comprobar desequilibrios en el mercado de trabajo comunitario.

Para poder estudiar la evolución de la inmigración en España en el último decenio, hay que hacer referencia a distintas fuentes de información estadística disponibles. El padrón municipal de habitantes, el censo de población y viviendas, las estadísticas de permisos de residencia y la encuesta de población activa son las fuentes principales que, en estos momentos, utilizamos para estimar el número de migrantes en España y sus características sociodemográficas. Sin embargo, estas fuentes tienen una serie de limitaciones que no me resisto a enunciar. Éstas son:

- La cifra real de población extranjera y migrante en España es mayor que la contabilizada. Esta subestimación puede ser aún más grave si atendemos a criterios de procedencia (es más difícil cuantificar a los asiáticos y africanos que a los europeos o americanos) y a tiempo de residencia. ¿Cuál es el volumen de la migración irregular en España?

- Por otro lado, las estadísticas no han tomado en cuenta los procesos extraordinarios de regularización lo que hace cuestionar la evolución numérica de los migrantes, especialmente en los últimos tres años.

Sin embargo, todos los investigadores parecen coincidir que las distintas fuentes empleadas sirven de aproximación a un fenómeno complejo que, evidentemente, es difícil de cuantificar precisamente por la naturaleza del mismo. Las características básicas de la población inmigrante en España son:

- Un peso muy fuerte de algunas nacionalidades. En particular, las sudamericanas (Ecuador) y las norteafricanas (Marruecos). Sin embargo, se ha empezado a detectar un incremento importante en otros grupos, concretamente en el caso de ciudadanos de países fuera del ámbito de la UE.

- Una población relativamente joven (entre 15 y 40 años) que presenta un equilibro casi completo entre hombres y mujeres salvo en algunos grupos de países de origen. Estos grupos de edades son los conside- 
rados en edad de trabajar y económicamente activos. Además, se da una importante diversidad en las formas de convivencia así como en la composición de los hogares, incrementándose significativamente el número de parejas con hijos. En el caso del Ecuador, en 2003 las mujeres representaban el $51,2 \%$ del total de ecuatorianos empadronados en España, las colombianas el 57\% y las peruanas el 57,2\%.

Tabla 1. Principales nacionalidades en la población inmigrante empadronada, 2003

\begin{tabular}{l|c|c|} 
PAÍS & POBLACIÓN & \% SOBRE POBLACIÓN NO COMUNITARIA \\
Ecuador & 390.000 & 18,7 \\
Marruecos & 379.000 & 18,2 \\
Colombia & 244.700 & 11,7 \\
Rumanía & 137.300 & 6,6 \\
Argentina & 109.400 & 5,2 \\
Perú & 55.900 & 2,7 \\
Bulgaria & 52.800 & 2,5 \\
China & 51.200 & 2,5 \\
Total no UE & 2.085 .300 & 68,1
\end{tabular}

Fuente: INE, Padrón Municipal de habitantes.

- Respecto a los niveles de estudio terminados, las diferentes fuentes de información revelan niveles similares a los de los españoles, e incluso mayor en ciertos grupos nacionales, exceptuando los africanos. Hay que tener presente que los datos sobre nivel de estudios se basan en datos subjetivos, en las declaraciones de los propios migrantes, cuestión que complica un poco la comparabilidad de los datos respecto a los españoles. Además, los sistemas educativos y las titulaciones oficiales no exactamente los mismos en España que en otros países y lo más importante, sin homologación oficial, las atribuciones profesionales de las titulaciones obtenidas en el extranjero no son reconocidas legalmente.

- Finalmente, atendiendo a los tiempos medios de residencia declarada, se observa una elevada frecuencia de asentamiento en España, al menos en lo que se refiere a los que han alcanzado el medio plazo. Sin embargo, estos datos no reflejan exactamente el grado de asentamiento y de no retorno de los migrantes, cuestión que retomaré más tarde.

\section{INMIGRACIÓN EN EL MERCADO DE TRABAJO ESPAÑOL}

En los últimos seis años la economía española ha ido creciendo a un ritmo superior que el de la media europea. La inflación se ha mantenido a niveles aceptables de convergencia europea y el déficit público y su reducción ejemplar se ha tomado como modelo para las economías europeas. Sin embargo, los beneficios del crecimiento económico se han 
concentrado en sectores empresariales muy concretos, en la banca y la riqueza se ha concentrado de manera notable. Los efectos redistributivos de un sistema fiscal cada vez más regresivo y la reducción del gasto público (en particular del gasto social) ha afectado a esta polarización de los beneficios. El desmantelamiento del Estado de Bienestar español, primero con el retroceso de la inversión en servicios sanitarios y sociales y después con la privatización de la gestión ha tenido efectos ciertamente sobre la población general.

En España se ha creado un notable número de puestos de trabajo, se han destruido casi la mitad de éstos, se ha precarizado por ley la contratación y se han vulnerado los derechos de los trabajadores flexibilizando el despido, que casi no tiene consecuencias monetarias para los empleadores (tanto públicos como privados).

Un sistema de solidaridad intergeneracional, basado en las contribuciones a través del trabajo a medio y largo plazo, no puede sostener sin inversión pública el régimen de pensiones y jubilaciones actuales, pero parece que eso queda solucionado con planes de pensiones privados que sólo puede pagar un cuarto de la población trabajadora. Si a esto le sumamos el precio de la vivienda (en la que un español medio invierte casi los dos tercios de su renta cuando pretende que sea en propiedad, o paga arrendamientos que se han incrementado notablemente en las grandes ciudades, incluidas sus periferias) podemos entender que las condiciones de vida en España no necesariamente han mejorado al ritmo que sí lo ha hecho la economía.

Sin embargo, la situación económica, política y social de otros países no comunitarios ha sido mucho peor en estos diez años. No se emigra sin motivo. Las expectativas de mejora, ajustadas a la realidad o construidas en el imaginario colectivo, hacen que nos movamos de un lugar a otro, del pueblecito a la ciudad, de una periferia a un centro, de una semiperiferia a otra, de una provincia como el Azuay (Ecuador), a otra como Alicante (España).

En estos seis años, lo que se van encontrando los emigrantes en lo tocante al mercado de trabajo en España es, a grandes rasgos y según los informes del Consejo Económico y Social, lo siguiente:

- Bajos niveles de participación laboral y elevados niveles de desempleo. En particular, entre población femenina y jóvenes (16 a 29 años) de ambos sexos.

- Altas tasas de temporalidad y elevados índices de rotación en el empleo, paro e inactividad, que afectan más a mujeres y a jóvenes. 
- Alta presencia de economía sumergida o irregular o informal que se concentra en sectores productivos concretos y en ciertas áreas geográficas en el territorio español. Es el caso de la provincia de Alicante o de Murcia, provincias que concentran un porcentaje elevado de los migrantes ecuatorianos en España.

- Bajo crecimiento de la productividad del trabajo, como consecuencia de una estructura productiva compuesta por pequeñas empresas en las que las actividades intensivas en mano de obra siguen siendo predominantes.

- Baja movilidad de la población activa que se combina con las diferencias territoriales respecto a mercado de trabajo.

\section{FACTORES DE INCLUSIÓN Y EXCLUSIÓN SOCIAL DE LOS MIGRANTES LATINOAMERICANOS EN ESPAÑA}

Si estas son las características del mercado de trabajo español, es fácil vincular la inserción de los migrantes latinoamericanos en los segmentos aún menos protegidos, siendo sistemáticamente explotados al conjugarse la irregularidad en términos legales con un mercado de trabajo polarizado y sin acceso real a puestos de trabajo bien remunerados.

Pero los procesos de inclusión/exclusión no sólo son apreciables en lo tocante al mercado de trabajo y a la actividad económica. Si bien el trabajo remunerado regularizado posibilita la satisfacción de las necesidades en el mercado a través del intercambio monetario y además contribuye al sostenimiento de las economías de los países emisores (como en el caso del Ecuador), es tan sólo un factor más en lo tocante a la participación de los beneficios que genera una sociedad y que deberían ser universales.

El Estado de Bienestar español, el Welfare del sur, que nunca existió en términos socialdemócratas europeos en España, ha generado sus propios excluidos. Creo que no es beneficioso para nadie estereotipar como excluidos y/o pobres a los migrantes latinos en España. Sin embargo, y por las cuestiones que he mencionado con anterioridad, sí es verdad que son personas con una altísima vulnerabilidad social.

A modo de síntesis y conclusión, mostraros algunos elementos para el debate posterior, relacionados estos factores de inclusión/exclusión con la clara vocación política de facilitar las mejores condiciones de vida a toda la población que habita en un territorio. 
Cuadro 2. Factores de inclusión/exclusión de los migrantes latinoamericanos en España

\begin{tabular}{|c|c|c|}
\hline & FACTORES DE INCLUSION & FACTORES DE EXCLUSION \\
\hline $\begin{array}{l}\text { Economía } \\
\text { Mercado de trabajo }\end{array}$ & $\begin{array}{l}\text { - Fácil empleabilidad } \\
\text { - Acceso rápido al empleo } \\
\text { - Remesas }\end{array}$ & $\begin{array}{l}\text { - Empleos en el mercado secundario } \\
\text { de trabajo } \\
\text { - Economía informal } \\
\text { - No ahorro }\end{array}$ \\
\hline Política & - Acuerdos bilaterales entre Estados & - Política de cupos \\
\hline Legalidad & - Periodos de regularización & - Racismo institucional \\
\hline Derechos & - Nacionalidad (arraigo) & - No participación política \\
\hline $\begin{array}{l}\text { Sistema educativo } \\
\text { Cultura }\end{array}$ & $\begin{array}{l}\text { - Titulaciones } \\
\text { - Misma lengua } \\
\text { - "Tradición" }\end{array}$ & $\begin{array}{l}\text { - No homologación de títulos } \\
\text { - Dificultades lingüísticas } \\
\text { - "Tradición" } \\
\text { - "Ghettos" culturales }\end{array}$ \\
\hline Relaciones sociales & $\begin{array}{l}\text { - Redes sociales de compatriotas } \\
\text { (no asociaciones) } \\
\text { - ONG's locales } \\
\text { - Asociaciones de migrantes } \\
\text { - Reagrupamiento familiar }\end{array}$ & $\begin{array}{l}\text { - Ruptura familiar } \\
\text { - Etiquetaje social } \\
\text { - Desconfianza en las asociaciones } \\
\text { de migrantes }\end{array}$ \\
\hline
\end{tabular}

Frente al actual modelo de desarrollo capitalista, centrado en el crecimiento económico abstracto, basado, a su vez, en la competitividad internacional y en las oportunidades que parece ofrecer la globalización para la integración de los mercados mundiales y que condiciona los planes de vida de más $80 \%$ de la población mundial, se deben plantear modelos de desarrollo endógenos que se centren en la satisfacción de las necesidades humanas básicas de la población cooperando, no compitiendo, respetando el medioambiente y no depredando, organizando la actividad económica lejos de los principios de la modernidad rostowniana. Hay experiencias. No hay mucho que inventar. Quizá si nos ponemos de acuerdo en ayudarnos compartiendo y en no permitir la polarización de la riqueza y la pobreza, ni siquiera por parte de los Estados, los millones de personas que se desplazan como migrantes, con sus historias de vida, con todos los factores subjetivos que queramos y que debemos estudiar como hacemos, tendrían otras expectativas vitales.

El análisis de la realidad deja, siempre que se hace con cierta sensibilidad por las personas con las que compartimos planeta, un sabor agridulce de historias de maldesarrollo pero también de intentos de cambiar situaciones muy duras. Nuestros migrantes españoles de los años 60 a Europa dan fe. También nuestros migrantes lationamericanos actuales. Cómo trabajar en proyectos de desarrollo atravesados por movilidad humana es algo que nos involucra. Desde ahora mismo. En todo proceso de cambio hay reminiscencias de los tiempos pasados. Yo creo que no sólo el volumen de las migraciones internacionales evidencia un cambio en el sistema mundial sino más bien la preocupación por trabajar por sociedades más libres, menos alienadas, más cooperativas, menos extrañas, es decir, más desarrolladas. 


\section{BIBLIOGRAFÍA}

APARIClO, R. La inmigración y la economia española. Rosa Aparicio y Andrés Tornos. Madrid: Ministerio de Trabajo y Asuntos Sociales, Secretaría General de Asuntos Sociales, Instituto de Migraciones y Servicios Sociales, 2000. - 181 p.

BONELLI JÁUDENES, E. Tráfico e inmigración de mujeres en España: colombianas y ecuatorianas en los servicios domésticos y sexuales. Elena Bonelli Jáudenes y Marcela Ulloa Jiménez. Madrid: Acsur-Las Segovias, 2001. - 121 p.

BORREGO VEGA, A.L. Mujer y migración: alcances de un fenómeno nacional y regional. Quito: Instituto Latinoamericano de Investigaciones Sociales (ILDIS); Abya-Yala, [1995?]. - $116 \mathrm{p}$.

CACHÓN RODRIGUEZ, L. Prevenir el racismo en el trabajo en España. Lorenzo Cachón; con la colaboración de Rocío Moldes; prólogo de Antonio Gutiérrez. Madrid: Ministerio de Trabajo y Asuntos Sociales. Instituto de Migraciones y Servicios Sociales, 1999, - 291 p.

Colectivo IOÉ. Exploración bibliográfica sobre Ecuador y la situación de inmigrantes ecuatorianos en España. Colectivo Ioé; elaborado por Raquel Mallouh Verdeal. Informe 3. Línea 6: Investigación Migración, comunicación y desarrollo Ecuador-España. 2001, 24 p.

Colectivo IOÉ. Inmigrantes, trabajadores, ciudadanos : una visión de las migraciones desde España. Valencia : Patronat Sud-Nord de la Universitat de València, 1999. - 258 p.

Colectivo IOÉ. Las remesas de los inmigrantes Ecuatorianos. Funcionamiento y características de las Agencias de envío de dinero en España. Informe 1. Línea 6: Investigación Migración, comunicación y desarrollo Ecuador-España. 2001.18 p.

Colectivo IOÉ. Los residentes ecuatorianos en España. Informe 2. Línea 6: Investigación Migración, comunicación y desarrollo Ecuador-España. 2001.21 p.

FISAS, V. Las migraciones: el olvido de muestra historia. Zaragoza : Seminario de Investigación para la Paz, 1994. - $112 \mathrm{p}$.

HERRANZ GÓMEZ, Y. Formas de incorporación laboral de la inmigración latinoamericana en Madrid: importancia del contexto de recepción [Tesis de doctorado]. Yolanda Herranz. Gómez; Ubaldo Martínez Veiga (dir.). 1996. - 463 p.

Madrid : Servicio de Publicaciones de la OEI, 1988. - 173 p.

MARRODAN, M.D. El asociacionismo iberoamericano en Madrid. M.D. Marrodán, Y. Herranz y C.M. Garcia Ruiz. [Madrid : Asociación Madrileña de Antropología, 1991]. - [12] h.

MARTÍNEZ VEIGA, U. La integración social de los inmigrantes extranjeros en España. Madrid: Trotta, 1997. - $299 \mathrm{p}$.

MARTÍNEZ VEIGA, U. Pobreza, segregación y exchisión espacial: la vivienda de los inmigrantes extranjeros en España. Barcelona: Icaria, D.L. 1999. - 175 p. 